\title{
Resonant Inverse Compton Scattering above Polar Caps: Gap Acceleration Efficiency for Young Pulsars
}

\author{
Qinghuan Luo and R. J. Protheroe \\ Department of Physics and Mathematical Physics, \\ University of Adelaide, Adelaide, SA 5005, Australia \\ qluo@physics.adelaide.edu.au \\ Received 1997 August 1, accepted 1997 November 18
}

\begin{abstract}
It is shown that for moderately hot polar caps (with effective temperature of $\sim 10^{6} \mathrm{~K}$ ), the efficiency of polar gap acceleration is lower compared to the case in which the polar caps are relatively cool and inverse Compton scattering plays no role in controlling the gap. For young pulsars with superstrong magnetic fields $\left(\geq 10^{9} \mathrm{~T}\right)$ and hot polar caps (with temperature of $\geq 5 \times 10^{6} \mathrm{~K}$ ), because of the energy loss of electrons or positrons due to resonant inverse Compton scattering in the vicinity of polar caps, pair cascades occur at distances further away from the polar cap, and in this case we have a relatively high acceleration efficiency, with ions carrying most of the particle luminosity.
\end{abstract}

Keywords: acceleration of particles — pulsars: general — stars: neutron — scattering

\section{Introduction}

The mechanism for converting rotational energy of pulsars to electromagnetic radiation has long been the subject of active research in pulsar physics. The recent observation of seven pulsars by the instruments aboard CGRO provides even more challenge to the current pulsar theory. These pulsars radiate high-energy photons with apparently high efficiency (Ulmer 1994; Thompson et al. 1994). One possible way to convert the rotational energy to electromagnetic radiation is through particle acceleration by the rotation-induced electric field in the pulsar's magnetosphere. There are several sites for gap acceleration, which include the polar gap (Ruderman \& Sutherland 1975; Arons \& Scharlemann 1979; Michel 1974; Fawley, Arons \& Scharlemann 1977), the slot gap (Arons 1983), and the outer gap (Cheng, Ho \& Ruderman 1986). In the polar gap model, the acceleration region is located near the polar cap, while in the outer gap model, the acceleration occurs near the light cylinder. In the slot gap model, the acceleration is at the boundary between the open and closed field lines.

There is observational evidence that polar caps of young pulsars can be hot, with effective temperatures $T \sim 10^{6} \mathrm{~K}$ (Ögelman 1991; Greiveldinger et al. 1996). Although the exact value of $T$ is rather uncertain, and may depend on the model of the atmosphere above the polar cap (Romani 1987), the hot polar cap appears to be a plausible consequence of polar cap heating as the result of particle acceleration (Cheng \& Ruderman 1977; Arons \& Scharlemann 1979; Luo 1996). One of the important consequences of hot polar caps is that they provide an alternative mechanism for controlling the gap, i.e. mainly through pair cascades initiated by Comptonscattered photons (Sturner 1995; Luo 1996). Inverse Compton scattering in the polar cap region is strongly modified by the magnetic field in that the scattering cross section is enhanced by the cyclotron resonance (Herold 1979; Xia et al. 1985), i.e. in the electron rest frame, soft photons have energies close to the cyclotron energy $\varepsilon_{B}=B / B_{c}$ in $m_{e} c^{2}$, with $B_{c} \approx 4 \cdot 4 \times 10^{9} \mathrm{~T}$ the critical field. This process is referred to as resonant inverse Compton scattering (RICS). Because of the resonance, the effective cross section is greatly enhanced in comparison with the ordinary Compton scattering. For gap acceleration, we can define its efficiency as the ratio of the total voltage across the gap to the maximum voltage across the polar cap (i.e. for an empty magnetosphere). The efficiency of the gap acceleration is controlled by the pair production and thus strongly depends on the effective temperature of the polar caps.

In this paper, we consider the constraint imposed by RICS on the efficiency of polar gap acceleration. We assume that free emission of charges from polar caps is allowed, and consider both the polar region in which the outflowing charges are electrons $(\mathbf{B} \cdot \boldsymbol{\Omega}>0)$ and that in which the outflowing charges are heavy ions or positrons $(\mathbf{B} \cdot \boldsymbol{\Omega}<0)$. In the polar gap at which $\mathbf{B} \cdot \boldsymbol{\Omega}<0$, since the energy loss of ions due to RICS is negligibly small, they can be accelerated by the full potential drop across the gap. We derive the condition for the ions to carry most of the particle luminosity. Accelerated ions may produce 
pairs in the anisotropic thermal photon field. We calculate numerically the distance from the polar cap at which the ions start to produce pairs, and compare it to the gap length constrained by pair production by RICS.

\section{Limits on Particle Energies by Resonant Inverse Compton Scattering}

For a hot polar cap that emits soft photons, RICS can constrain the maximum energies of electrons (or positrons) accelerated in the polar gap in the following two ways: the energy loss competes with acceleration, or the acceleration length is limited through pair production. For a given accelerating potential, $\phi(x)$, the Lorentz factor of an electron (or positron) at a distance $x R_{0}$ to the polar cap is given by (Dermer 1990; Luo 1996)

$$
\begin{aligned}
\gamma(x)= & \gamma_{0}+\frac{e}{m_{e} c^{2}} \phi(x) \\
& -\frac{R_{0} \sigma_{T}}{\lambda_{c}^{3}} \frac{\Theta}{\pi^{2} \alpha_{f} \beta} \int_{0}^{x} d x^{\prime}\left[\frac{\xi\left(x^{\prime}\right) \varepsilon_{B}^{2}}{\gamma\left(x^{\prime}\right)}\right],
\end{aligned}
$$

where $\gamma_{0}$ is the initial Lorentz factor, $\lambda_{c}=$ $\hbar / m_{e} c$ is the Compton wavelength, $\Theta=1.686 \times$ $10^{-4}\left(T / 10^{6} \mathrm{~K}\right)$ is the effective temperature (in $\left.m_{e} c^{2}\right), R_{0}$ is the star's radius, $\sigma_{T}$ is the Thomson cross section, $\alpha_{f} \approx 1 / 137$ is the fine-structure constant, $\xi\left(x^{\prime}\right)=\varepsilon_{B} /\left[\gamma\left(x^{\prime}\right)\left(1-\beta\left(x^{\prime}\right) \cos \theta_{\max }\right)\right]$, and $\theta_{\max }$ is the maximum angle between a photon and the particle motion. For thermal emission from the entire neutron star's surface we have $\theta_{\max }=\operatorname{arcos}\left\{\left[(x+1)^{2}-1\right]^{\frac{1}{2}} /(x+1)\right\}$, which corresponds to propagation of photons at directions tangential to the surface. For a hot polar cap, this is valid only for $x<0.5 \theta_{c}^{2}$ [where $\theta_{c}=\left(2 \pi R_{0} / P c\right)^{\frac{1}{2}}$ and $P$ is the pulsar period]; for $x>0 \cdot 5 \theta_{c}^{2}$, the angle is given by $\theta_{\max } \approx \operatorname{arcos}\left[x /\left(\theta_{c}^{2}+x^{2}\right)^{\frac{1}{2}}\right]$, corresponding to photons from the polar cap rim on which the field lines have colatitude angle $\theta_{c}$ relative to the magnetic pole. The energy loss due to RICS is described by the third term on the right-hand side of equation (1).

The maximum Lorentz factor corresponds to the value at $x=x_{\max }$, which is the gap length determined by the pair production, e.g. due to RICS or due to thermal photons interacting with heavy ions, whichever comes first. Equation (1) can be solved numerically with the boundary condition $\gamma(0)=\gamma_{0}$.

The model for the accelerating potential strongly depends on the physical conditions on the polar cap. If polar caps are sufficiently hot, we may have free emission of charges. The induced electric field causes charge flow, which tends to screen out the field. However, the charge screening is always insufficient, e.g. due to field line curvature (Arons \& Scharlemann 1979), and hence charge depletion occurs [the charge density deviates from the Goldreich-Julian (GJ) density]. An electric potential exists in the charge-depletion region (often called the gap). As an example, we consider the potential (e.g. Arons \& Scharlemann 1979; Arons 1983)

$$
\begin{aligned}
\phi(x) \approx & 0 \cdot 375 \theta_{c}^{4} c B R_{0}\left(\frac{\theta}{\theta_{c}}\right)\left[1-\left(\frac{\theta}{\theta_{c}}\right)^{2}\right] \\
& \times g(x) \sin \psi \sin i,
\end{aligned}
$$

where $\theta$ is the angle of the field lines relative to the magnetic pole, $\psi$ is the azimuthal angle of the open field line flux tube, $x$ is the distance to the polar cap (in $R_{0}$ ), $i$ is the angle between the rotation axis and the magnetic pole, and $g(x)$ is given by $g(x)=x^{2}$ for $x<0 \cdot 5 \theta_{c}$, $g(x)=\theta_{c}\left[(x+1)^{\frac{1}{2}}-1\right]$ for $x \gtrsim 0 \cdot 5 \theta_{c}$. According to Arons (1983), the angle $\psi$ is in the range $-\pi \lesssim \psi \lesssim 0$ for $i>\pi / 2$ (if the outflowing charges are ions), and $0 \lesssim \psi \lesssim \pi$ for $i<\pi / 2$ (if the outflowing charges are electrons). The potential given by equation (2) is due to charge density decrease along field lines (i.e. it becomes less than the GJ density) because of field line curvature. Plots of the Lorentz factor as a function of distance from the polar cap are shown in Figure 1. We assume $\gamma=10$ at $x R_{0}=1 \mathrm{~cm}$. As shown in the figure, energy loss due to RICS can compete with acceleration only for a high polar cap temperature $T \gtrsim 5 \times 10^{6} \mathrm{~K}$. For $T \approx 10^{6} \mathrm{~K}$, the particle energy is constrained mainly through pair production by RICS.

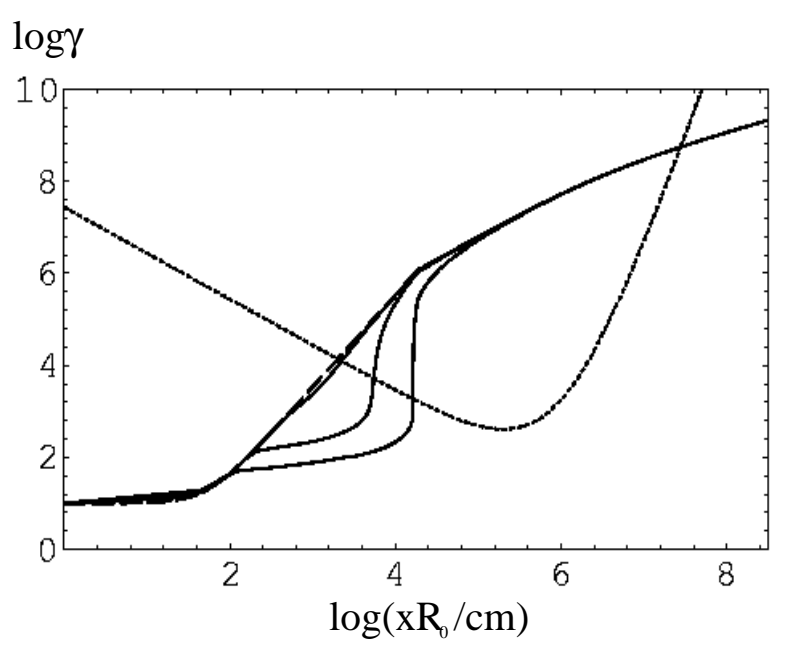

Figure 1-Lorentz factor versus distance to the polar cap. The electrons (or positrons) are accelerated by the potential (2) with $i=\pi / 4$ (or $3 \pi / 4$ ), $B=3 \times 10^{9} \mathrm{~T}, P=0 \cdot 15 \mathrm{~s}$. The dashed curve is obtained without RICS. The solid curves (from top to bottom) correspond to $T=1.5 \times 10^{6} \mathrm{~K}$, $5 \times 10^{6} \mathrm{~K}$, and $10^{7} \mathrm{~K}$. We assume that thermal emission is from the polar cap. The dotted curve is the threshold condition for pair production through RICS (i.e. pair production can occur only above the curve).

There are other models of the space-charge-limited potential, such as the model discussed by Michel (1974) and also by Fawley, Arons \& Scharlemann 
(1977) and Cheng \& Ruderman (1977), which do not rely on field line curvature and in general give a lower voltage. The potential discussed by Michel (1974) arises from the deviation of the charge density from the GJ value because of particle inertia, i.e. the velocity $v(x)$ and the magnetic field $B(x)$ have different scaling with $x$, such that the charge density cannot be maintained at the GJ density all the way along open field lines. The corresponding potential can be described by (Fawley, Arons \& Scharlemann 1977)

$$
\phi(x)=2 x c B R_{0}\left(\frac{\Omega}{\Omega_{B}}\right)^{\frac{1}{2}}
$$

with $\Omega=2 \pi / P, \Omega_{B}=Z e B / m$ and $x \lesssim \theta_{c}$. For $x>\theta_{c}$, the electric field drops off faster than $\left(x R_{0}\right)^{-2}$. The plot of the Lorentz factor as a function of $x R_{0}$ is shown in Figure 2 for $T=1.5 \times 10^{6}$ and $T=5 \times 10^{6}$. The electric potential for $x>\theta_{c}$ is assumed to be constant, $\phi(x)=\phi\left(\theta_{c}\right)$. For $T=5 \times 10^{6} \mathrm{~K}$, the energy loss is so severe that it continues beyond the distance $x>\theta_{c}$, resulting in a sudden decrease in $\gamma$ at $x=\theta_{c}$. As in Figure 1, for moderately hot polar caps $\left(T \approx 10^{6} \mathrm{~K}\right)$, the particle energy is constrained by electron-positron cascades started by RICS.

\section{$\log \gamma$}

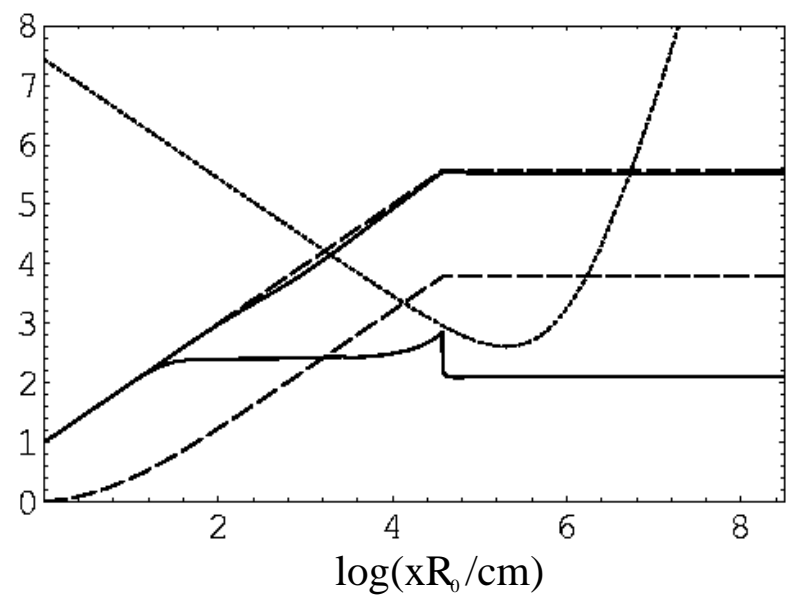

Figure 2-As in Figure 1 but the particles are accelerated by the potential (3) with $i=0(i=\pi$ for outflowing positrons or ions), $P=0 \cdot 15 \mathrm{~s}, B=3 \times 10^{9} \mathrm{~T}, T=1.5 \times 10^{6} \mathrm{~K}$ (upper solid curve) and $5 \times 10^{6} \mathrm{~K}$ (lower solid curve). The result is similar to that derived by Sturner (1995). The two dashed plots (upper and lower) correspond respectively to acceleration of electron (positron) and ion without RICS. The dotted curve is the threshold condition for pair production through RICS.

The main mechanism for a high-energy photon (emitted by an electron or positron either through curvature radiation or inverse Compton scattering) to produce a pair is single photon decay in a strong magnetic field. To produce a pair, the photon must satisfy the threshold condition $0 \cdot 5 \varepsilon \sin \theta_{\gamma} \gtrsim 1$, where $\varepsilon$ is the photon energy (in $m_{e} c^{2}$ ), and $\theta_{\gamma}$ is the angle between the photon propagation and the magnetic field. When this condition is satisfied, the number of pairs produced depends on the opacity, $\tau$, which is an integration of the absorption coefficient (for a photon being converted into a pair) along the distance that photon travels. We assume that a photon produces at least one pair if $\tau \gtrsim 1$. With this condition, we can write down the threshold condition for producing at least one pair through RICS (Luo 1996) as

$$
\begin{aligned}
& \gamma> 13 \cdot 4\left(\frac{15}{\ln \Lambda}\right)\left(\frac{B}{3 \times 10^{9} \mathrm{~T}}\right)^{-2} \\
& \times\left(\frac{P}{0 \cdot 1 \mathrm{~s}}\right)^{\frac{1}{2}} \frac{(1+x)^{6}}{x}
\end{aligned}
$$

The right-hand side of equation (4) strongly depends on $B$. If the above condition is satisfied, pair cascades can be initiated by RICS. The threshold condition is represented by the dotted curves in Figures 1 and 2 . Note that for each curve of $\log \gamma$ vs $\log \left(x R_{0} / \mathrm{cm}\right)$, the location of an intersection (with the dotted curves) point defines a gap length, represented by $x_{0}^{\text {RICS }}$. (If the curve has two intersecting points, the one that is the nearest to the polar cap is relevant.)

\section{Pair Production by Photons in Field of Nucleus}

Electron-positron pair cascades can be started by accelerated ions in thermal photon fields. A photon can decay into an electron-positron pair in the Coulomb field of a nucleus when the centre of momentum frame energy exceeds the rest mass of the nucleus plus two electrons, that is

$$
s>\left(m_{N} c^{2}+2 m_{e} c^{2}\right)^{2},
$$

where $m_{N}$ is the mass of the nucleus. This condition can be satisfied for soft photons from the polar cap in the presence of accelerated ions. We can estimate the mean free path of an ion before producing an average of one pair. If we believe one pair per primary particle is enough to short out the electric field, this mean free path length, represented by $x_{0}^{\text {ion }}$, can be identified as the gap length (Figures 3 and 4).

Numerical evaluation of the mean free path for an accelerated ion traversing an isotropic thermal photon field was given by Protheroe (1984) and Protheroe \& Johnson (1996). The injection rate of pairs for a given energy of iron nuclei was numerically calculated by Bednarek \& Karakula (1995) for an anisotropic photon field from the polar cap. We calculate the number of pairs produced by iron nuclei accelerated along open field lines by the potentials given by equations (2) and (3), and the mean free 
path corresponds to the average distance at which one pair is produced per iron nucleus. The mean cumulative number of pairs produced in the radiation field of the polar cap (or whole neutron star) at temperature $T$ by a nucleus of energy $E$ traveling along the neutron star magnetic axis from the pole to distance $x R_{0}$ above the polar cap surface is given by

$$
\begin{aligned}
N_{e^{+} e^{-}}(E) \approx & \frac{R_{0}}{2} \int_{0}^{x} d x \int_{0}^{\infty} d \epsilon n(\varepsilon) \\
& \times \int_{\cos \left[\theta_{\max }(x)\right]}^{\cos \left[\theta_{\min }(E, \varepsilon)\right]} d \cos \theta(1-\beta \cos \theta) \sigma(s)
\end{aligned}
$$

where $n(\varepsilon)$ is the differential photon number density of photons of energy $\varepsilon$ in blackbody radiation at temperature $T$, and $\sigma(s)$ is the total cross section for pair production (Protheroe 1997) by a nucleus at centre of momentum (CM) frame energy squared, $s$, given by

$$
s=m_{N}^{2} c^{4}+2 \varepsilon E(1-\beta \cos \theta),
$$

where $\theta$ is the angle between the directions of the nucleus and photon. Note that $\theta_{\min }(E, \varepsilon)$ is determined by the threshold condition (5). Plots of the accumulative number of pairs produced per ion through interaction with thermal photons as a function of distance from the polar cap are shown in Figure 4 for $P=0 \cdot 15 \mathrm{~s}$ and $B=3 \times 10^{9} \mathrm{~T}$. Compared to RICS, in this case we have $x_{0}^{\text {RICS }}<x_{0}^{\text {ion }}$. Although we use a high $B$, the process strongly depends on $P$ and $T$.

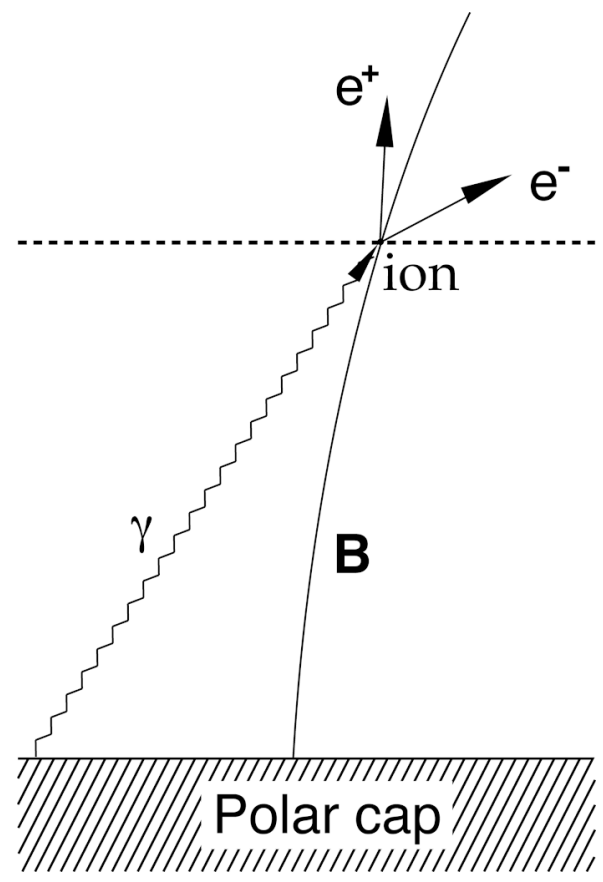

Figure 3-Pair production due to an ion colliding with a soft photon from polar cap emission above the polar cap with $\boldsymbol{\Omega} \cdot \mathbf{B}<0$. Besides ions, the outflowing charges may include positrons.

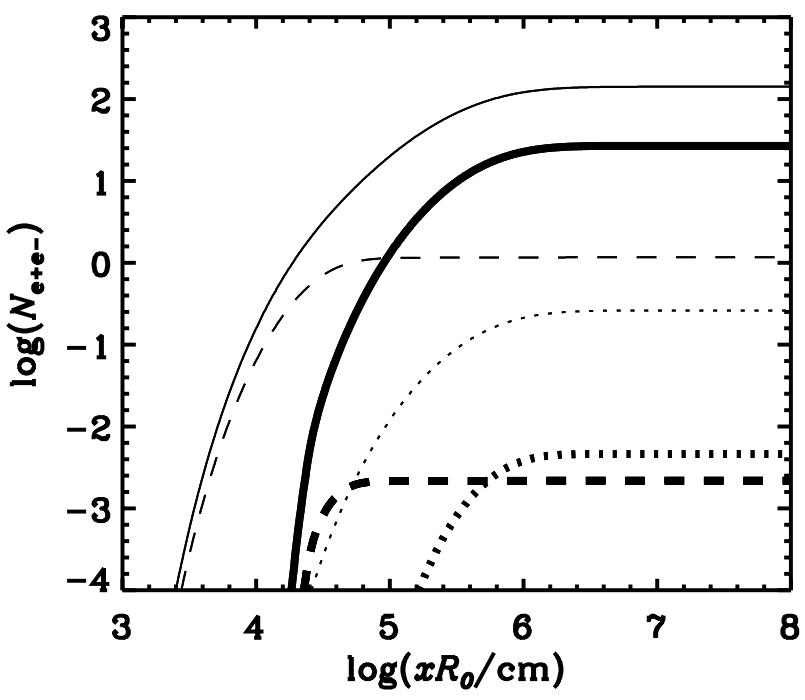

Figure 4-Plots of the cumulative number of pairs produced per ion as a function of distance. Solid, dashed and dotted curves correspond respectively to $T=5 \times 10^{6} \mathrm{~K}$ for the whole star's surface, $T=10^{7} \mathrm{~K}$ for the polar cap (but the star's surface has $T=10^{6} \mathrm{~K}$ ), and $T=5 \times 10^{6} \mathrm{~K}$ for the polar cap (but the star's surface has $T=5 \times 10^{5} \mathrm{~K}$ ). The thick and thin curves are calculated using the potentials (2) and (3), respectively. We assume $P=0 \cdot 15 \mathrm{~s}, B=3 \times 10^{9} \mathrm{~T}$.

\section{Acceleration Efficiency}

The efficiency of the polar gap is described by the ratio of the potential drop across the gap, $\phi\left(x_{0}\right)$, to the maximum potential across the polar cap with an empty magnetosphere, $\phi_{\max }$, viz.

$$
\eta=\frac{\phi\left(x_{0}\right)}{\phi_{\max }},
$$

where $\phi_{\max }=0.5 \theta_{c}^{4} c B R_{0} \approx 6.6 \times 10^{12} \mathrm{~V}(1 \mathrm{~s} / P)^{2}$ $\left(B / 10^{8} \mathrm{~T}\right), \theta_{c}=\left(\Omega R_{0} / c\right)^{\frac{1}{2}}=1.45 \times 10^{-2}(1 \mathrm{~s} / P)^{\frac{1}{2}}$, and $x_{0}$ is the length of the polar gap in units of $R_{0}$. Let $L_{\mathrm{p}}$ and $L_{\mathrm{R}}$ be the accelerated particle and spin-down luminosities, respectively. If the energy loss of accelerated particles in the gap is not important, we have $\eta \approx L_{\mathrm{p}} / L_{\mathrm{R}}$, since we can write $L_{\mathrm{p}} \approx Z e \phi\left(x_{0}\right) \dot{N}_{\mathrm{GJ}}$ and $L_{\mathrm{R}} \approx Z e \phi_{\mathrm{max}} \dot{N}_{\mathrm{GJ}}$, where $\dot{N}_{\mathrm{GJ}}$ is the injection rate calculated from the GJ density $n_{\mathrm{GJ}} \approx 2 \epsilon_{0} B \Omega / Z e$. Note that the efficiency defined by equation (8) is similar to that used by Arons (1996). For the presently available models for accelerating potentials, we always have $\eta<1$.

Using equation (8), we may estimate the efficiency for a given model potential. For the potential described by equation (2) and assuming $x_{0} \gg 0 \cdot 5 \theta_{c}$, we have the maximum efficiency

$$
\eta_{\max } \approx 0 \cdot 29 \theta_{c} x_{0}^{\frac{1}{2}} \sin \psi \sin i
$$

Thus, even when we use $x_{0} \sim R_{L} / R_{0}=1 / \theta_{c}^{2}$, the maximum efficiency is $\eta_{\max } \lesssim 0 \cdot 29$. When a pair cascade occurs, we usually have $x_{0} \ll 1 / \theta_{c}^{2}$ (for the polar gap) and hence $\eta \ll \eta_{\max }$. 
One may estimate the efficiency for the potential given by equation (3) from

$$
\eta=0 \cdot 069\left(\frac{A}{Z}\right)^{\frac{1}{2}}\left(\frac{x}{\theta_{c}}\right)\left(\frac{10^{8} \mathrm{~T}}{B}\right)^{\frac{1}{2}}\left(\frac{P}{1 \mathrm{~s}}\right),
$$

with $x \lesssim \min \left\{\theta_{c}, x_{0}\right\}$. Although we have a higher $\eta$ for the Arons \& Scharlemann model, the acceleration by the potential given by equation (3) is the more effective for $i \approx 0$.

The evaluation of $x_{0}$ depends on the specific mechanism for initiating a pair cascade, and whether the pair plasma produced through the cascade is dense enough to short out part of the electric field. We consider three mechanisms for starting a pair cascade: RICS, curvature radiation, and inelastic scattering of ions by thermal photons from the polar cap. The corresponding lengths are $x_{0}^{\text {RICS }}, x_{0}^{\text {curv }}$ and $x_{0}^{\text {ion }}$. In general, we have $x_{0}^{\text {RICS }}<x_{0}^{\text {curv }}$ (e.g. Luo 1996). For moderately hot polar caps with effective temperatures of $\sim 10^{6} \mathrm{~K}$, the energy loss due to RICS is not important compared to acceleration but the photons produced through RICS can start a pair cascade at a distance less than $x_{0}^{\mathrm{RICS}}<x_{0}^{\text {curv }}$, $x_{0}^{\text {ion }}$. Thus, the effect of RICS is to reduce the gap acceleration efficiency. For space-charge-limited flow, the composition of outflowing charges at the poles with $\boldsymbol{\Omega} \cdot \mathbf{B}>0$ and $\boldsymbol{\Omega} \cdot \mathbf{B}<0$ can be different. For $\boldsymbol{\Omega} \cdot \mathbf{B}>0$, the primary particles consist mainly of electrons, and for $\boldsymbol{\Omega} \cdot \mathbf{B}<0$, the main components are heavy ions or positrons. In the ion zone, the gap length is constrained by the pair production by positrons through RICS. The possible source of positrons was discussed by Cheng \& Ruderman (1977). For moderately hot polar caps, the gaps at both types of pole have a similar efficiency.

For sufficiently hot polar caps and a superstrong magnetic field $\left(\sim 10^{9} \mathrm{~T}\right)$, the energy loss of electrons or positrons due to RICS can be important, and may prevent them starting a pair cascade in the region close to the polar cap. Since the cross section is $\propto\left(Z^{4} / A^{2}\right)\left(m_{e} / m_{p}\right)^{2} \approx 10^{-4}$ for iron nuclei $(Z / A \approx$ $1 / 2)$, the energy loss due to RICS for ions is negligibly small, and they can be continuously accelerated until distance $x \lesssim \min \left\{x_{0}^{\mathrm{RICS}}, x_{0}^{\mathrm{ion}}\right\}$. Thus, the acceleration efficiency increases significantly. As an example, for the potential (2) with $B=3 \times 10^{13}$ and $T=1.5 \times 10^{6} \mathrm{~K}$, the gap length is about $x_{0}^{\text {RICS }} R_{0} \approx 3 \times 10^{3} \mathrm{~cm}$ and the maximum energy of electrons (or positrons) is $10^{4} m_{e} c^{2}$. This gives the particle luminosity $L_{\mathrm{p}} \approx 10^{4} m_{e} c^{2} \dot{N}_{\mathrm{GJ}}$, where $\dot{N}_{\mathrm{GJ}}$ is the injection rate of primary electrons (or positrons). For hot polar caps with $T=10^{7} \mathrm{~K}$, from Figure 1, we obtain $x_{0}^{\text {RICS }} R_{0} \approx 1.6 \times 10^{4} \mathrm{~cm}$. From Figure 4 , pair production by ions interacting with thermal photons is important only for the case where the whole star's surface has $T=5 \times 10^{6} \mathrm{~K}$ (the thick solid curve), and this occurs at the distance $x_{0}^{\text {ion }} R_{0} \approx 10^{5} \mathrm{~cm}$.
Thus, the gap length is controlled by RICS. From Figure 1 , we have $\gamma\left(x_{0}^{\mathrm{RICS}}\right) \approx 1 \cdot 6 \times 10^{5}$ for positrons (or electrons) and $\gamma\left(x_{0}^{\mathrm{RICS}}\right) \approx\left(Z m_{e} / A m_{p}\right) \times 10^{6}$ for ions; positrons (or electrons) and ions can be accelerated to the maximum energy $1.6 \times 10^{5} m_{e} c^{2}$ and $10^{6} Z m_{e} c^{2}$, respectively. Hence, we have much a higher particle luminosity $L_{\mathrm{p}} \approx 10^{6} m_{e} c^{2} \dot{N}_{\mathrm{GJ}}$, and most of $L_{\mathrm{p}}$ is carried by ions.

\section{Application to Young Pulsars}

From observations, several pulsars may have polar caps with effective temperatures $\geq 10^{6} \mathrm{~K}$, though the exact value of the temperature is rather uncertain (for a review see e.g. Ögelman 1991). For moderately hot polar caps and typical $B$ fields $\left(B \sim 10^{8} \mathrm{~T}\right)$, the energy loss rate is not rapid enough to compete with the acceleration, and RICS controls the polar gap only through pair production. This in general reduces the gap length, and hence reduces the acceleration efficiency by at least one order of magnitude, imposing a severe constraint on the energetics of the polar gap.

For young pulsars with superstrong magnetic fields ( $\gtrsim 10^{9} \mathrm{~T}$ ), the gap acceleration efficiency can increase, provided that the polar cap $(\boldsymbol{\Omega} \cdot \mathbf{B}<0)$ is hot $\left(T \geq 5 \times 10^{6} \mathrm{~K}\right)$, and for electrons (or positrons), the energy loss due to RICS can dominate over the acceleration. One of the CGRO pulsars, PSR 1509-58, has magnetic field $\gtrsim 1.5 \times 10^{9} \mathrm{~T}$ (the magnetic field at the pole can be twice this value). Since neutron stars are hot at birth (after supernova explosion), their polar caps can be hot as a result of self-sustained polar cap heating at very young ages (e.g. Luo 1996), e.g. within 100 years or so after birth. These high-field pulsars, assuming they were born with high magnetic fields, must have undergone a phase when thermal emission had an important effect on pulsar electrodynamics.

We suggest that for high-field pulsars there can be four phases during their lifetime, as discussed below. (i) At very young age (within a few years after supernova explosion) when the surface or polar cap temperature is high, energy loss due to RICS is so severe that a pair cascade due to electrons or positrons cannot occur in the region close to the polar cap. In the ion zone $(\boldsymbol{\Omega} \cdot \mathbf{B}<0)$, accelerated ions may carry most of the particle luminosity. For heavy ions, photodisintegration may occur in the thermal photon field from the polar cap. At this stage, since pulsars also have very large spin-down luminosities, they are a potential source of high-energy hadrons and electromagnetic radiation. (ii) As pulsars cool down, the energy loss rate due to RICS becomes less than the acceleration rate. However, RICS may still be the dominant mechanism for pair production to constrain the gap length (Luo 1996). The high-field pulsar PSR 1509-58 may currently be at this stage, since the polar cap temperature inferred from the 
observation is about $1.5 \times 10^{6} \mathrm{~K}$ (e.g. Kawai et al. 1991). Its gap length is shorter than that controlled by pair production by either curvature radiation or relativistic ions in the photon field. Thus the acceleration efficiency is less than in phase (i). (iii) As the surface temperature or polar cap temperature becomes much lower, pair production by RICS is not important. The main mechanism for initiating pair cascades is curvature radiation. As the period increases, the gap length increases and hence the efficiency also increases. However, at this stage, the pulsar spin-down luminosity is lower than at a young age. (iv) The period reaches the critical period at which pair production due to curvature radiation is no longer operative. Although the gap acceleration efficiency is now high (no pairs to screen the electric field), the luminosity is very low.

In summary, for very young pulsars with superstrong magnetic fields $\left(B \gtrsim 10^{9} \mathrm{~T}\right)$ and hot polar caps $\left(\geq 5 \times 10^{6} \mathrm{~K}\right)$, the severe energy loss due to resonant inverse Compton scattering can prevent electrons or positrons from starting a pair cascade in the region close to the polar cap, but ions can be accelerated by the full potential drop across the gap (provided that free emission of ions can occur). We then have the ions carrying most of the particle luminosity. Thus very young pulsars with superstrong $B$ fields can be potential sources of high-energy hadrons and gamma-rays. In contrast, for pulsars with moderately hot polar caps $\left(T \sim 10^{6} \mathrm{~K}\right)$, the effect of resonant inverse Compton scattering is to reduce the gap acceleration efficiency. Pair production by accelerated ions interacting with thermal photons strongly depends on $P$ and $T$, and in general we have $x_{0}^{\text {RICS }}<x_{0}^{\text {ion }}$ and hence the polar gap length is controlled by RICS.

\section{Acknowledgments}

QL thanks the Australian Research Council (ARC) for financial support through a fellowship. We thank Wlodek Bednarek for helpful discussions.

\section{References}

Arons, J. 1996, A\&AS, 120, 49

Arons, J. 1983, ApJ, 266, 215

Arons, J., \& Scharlemann, E. T. 1979, ApJ, 231, 854

Bednarek, W., \& Karakula, S. 1995, Proc. 24th Int. Conf. on Cosmic Rays, Rome, Vol. 2, p. 279

Cheng, A. F., \& Ruderman, M. A. 1977, ApJ, 214, 598

Cheng, K. S., Ho, C., \& Ruderman, M. A. 1986, ApJ, 300, 500

Dermer, C. D. 1990, ApJ, 360, 197

Fawley, W. M., Arons, J., \& Scharlemann, E. T. 1977, ApJ, 217,227

Greiveldinger, C., et al. 1996, ApJ, 465, L35

Herold, H. 1979, Phys. Rev., D 19, 2868

Kawai, N., et al. 1991, ApJ, 383, L65

Luo, Q. 1996, ApJ, 468, 338

Michel, F. C. 1974, ApJ, 192, 713

Ögelman, H. 1991, in Neutron Stars: Theory and Observation, ed. J. Ventura \& D. Pines (Dordrecht: Kluwer), p. 87

Protheroe, R. J. 1984, Nature, 310, 296

Protheroe, R. J. 1998, in Towards the Millennium in Astrophysics: Problems and Prospects, ed. M. M. Shapiro \& J. P. Wefel (Singapore: World Scientific), in press

Protheroe, R. J., \& Johnson, P. A. 1996, Astroparticle Phys., 4, 253

Romani, R. W. 1987, ApJ, 313, 718

Ruderman, M., \& Sutherland, P. G. 1975, ApJ, 196, 51

Sturner, S. J. 1995, ApJ, 446, 292

Thompson, D. J., et al. 1994, ApJ, 436, 229

Ulmer, M. P. 1994, ApJSS, 90, 789

Xia, X. Y., Qiao, G. J., Xu, X. J., \& Hou, Y. Q. 1985, A\&A, 152, 93 